\title{
Boundary element dual reciprocity method with overlapping sub-domains
}

\author{
B. Thanh Tu \& V. Popov \\ Wessex Institute of Technology, Environmental Fluid Mechanics, \\ Ashurst Lodge, Southampton, UK
}

\begin{abstract}
In this paper a numerical approach based on the Boundary Element Method (BEM) with overlapping sub-domains is presented. The approach simplifies the assembly of the equations arising from the BEM sub-domain methods and at the same time reduces the problems with near-singular or singular integrals that may arise in the integral equations. The overlapping numerical approach is tested on a convection-diffusion problem. The approach is implemented in combination with the Dual Reciprocity Method (DRM) with two different Radial Basis Functions (RBFs) and the results are compared with the previous results obtained using the DRM-MD approach, showing good accuracy and convergence.
\end{abstract}

Keywords: boundary element method, domain decomposition, overlapping subdomains.

\section{Introduction}

The present formulation employs domain decomposition, but unlike the previous BEM sub-domain methods it applies an overlapping approach which eliminates the Interface Boundary Conditions (IBCs), simplifies assembly of the equations and reduces the problems arising from near-singular or singular integrals. It also leads to a closed system of equations while the BEM sub-domain techniques with continuous elements lead to over-determined systems.

When using domain decomposition in three dimensions (3D) and for the case where the number of sub-domains is large, imposing the IBCs may be complicated since several sub-domains would generally share the same node (vertex). In the BEM the IBCs include the flux conservation, which increases the 
complexity and therefore sometimes discontinuous elements are used in order to simplify the implementation of IBCs.

Another problem that often arises is the distortion in the geometry of some of the sub-domains due to complexity of the mesh. This may produce worsening of the condition number of the system of equations because of the proximity of the nodes and could introduce singularity or near-singularity in the integrals due to the distorted geometry of some of the sub-domains.

The proposed approach is quite general and can be applied to various BEM formulations, e.g., domain integration, DRM, multiple reciprocity method, etc. It eliminates the above mentioned problems due to IBCs and distorted sub-domains in the mesh.

\section{The boundary element dual reciprocity method}

In this section the boundary element dual reciprocity method is introduced though the approach will be effective with other BEM formulations as well. Let us consider the following equation:

$$
\nabla u^{2}(r)=b\left(r, u(r), \frac{\partial u(r)}{\partial x_{i}}, \frac{\partial u(r)}{\partial t}\right)
$$

where $u(r)$ is a potential field, $r$ is a position vector, $x_{i}$ is component of $r$, and $t$ is time. Given a point $r$ inside $\Omega$, by applying the Green formula to equation (1) in $\Omega$ bounded by $\Gamma$, (1) can be transformed into the following integral form:

$$
\lambda(r) u(r)+\int_{\Gamma} q^{*}(r, \xi) u(\xi) d \Gamma_{\xi}-\int_{\Gamma} u^{*}(r, \xi) q(\xi) d \Gamma_{\xi}=\int_{\Omega} u^{*}(r, \xi) b(\xi) d \Omega_{\xi}
$$

Here $u^{*}(r, \xi)$ is the fundamental solution of the Laplace problem which is given by

$$
u^{*}(r, \xi)=\frac{1}{2 \pi} \ln \left(\frac{1}{R}\right)
$$

for a $2 \mathrm{D}$ problems and

$$
u^{*}(r, \xi)=\frac{1}{4 \pi} \frac{1}{R}
$$

for 3D problems, where $R$ is the distance from the point of application of the concentrated unit source to any other point under consideration, i.e. $R=|r-\xi|$ and $q(\xi)=\partial u(\xi) / \partial n$ and $q^{*}(r, \xi)=\partial u^{*}(r, \xi) / \partial n$. The constant $\lambda(r)$ has value from 0 to 1 being equal to $1 / 2$ for smooth boundaries and 1 if the source point $r$ is inside the domain.

By applying the DRM approach as described in [1], one finally arrives at a boundary only integral representation formula

$$
\begin{aligned}
& \lambda(r) u(r)+\int_{\Gamma} q^{*}(r, \xi) u(\xi) d \Gamma_{\xi}-\int_{\Gamma} u^{*}(r, \xi) q(\xi) d \Gamma_{\xi}= \\
& \sum_{k=1}^{J_{b n}+J_{i n}}\left\{\alpha_{k}\left(\lambda(r) \hat{u}\left(r, \eta_{k}\right)+\int_{\Gamma} q^{*}(r, \xi) \hat{u}\left(\xi, \eta_{k}\right) d \Gamma_{\xi}-\int_{\Gamma} u^{*}(r, \xi) \hat{q}\left(\xi, \eta_{k}\right) d \Gamma_{\xi}\right)\right\}
\end{aligned}
$$


The DRM approximation employs $J_{b n}$ nodes on the boundary and $J_{i n}$ nodes inside the domain.

\subsection{Treatment of partial derivatives in the DRM}

In the classical DRM approach, in order to obtain expression for the partial derivatives, the potential at a point $r$ is expressed in terms of an approximation function $f$, in a similar way as it is done for $b[1]$

$$
u(\xi) \cong \sum_{k=1}^{J_{b n}+J_{i n}} f\left(\xi, \eta_{k}\right) \beta_{k}
$$

or expressed in matrix form:

$$
\mathbf{u}=\mathbf{F} \beta
$$

Differentiating both sides in (7) yields:

$$
\frac{\partial \mathbf{u}}{\partial x_{i}}=\frac{\partial \mathbf{F}}{\partial x_{i}} \beta
$$

From equation (7) the following expression can be derived $\beta=\mathbf{F}^{-1} \mathbf{u}$ which can be substituted into (8) producing:

$$
\frac{\partial \mathbf{u}}{\partial x_{i}}=\frac{\partial \mathbf{F}}{\partial x_{i}} \mathbf{F}^{-1} \mathbf{u}
$$

Recently Natalini and Popov [2] proposed another approach which has been implemented for 3D problems as well, which uses the normal derivative before the node $q_{i}^{b}$, and the normal derivative after the node $q_{i}^{a}$ to obtain the partial derivatives in the DRM-MD approach.

\section{Non-overlapping (classical) BEM sub-domain technique}

In this section the basic principles of the non-overlapping BEM sub-domain technique are introduced. In Figure 1 a case is presented where two sub-domains, 1 and 2, share a common interface where interface boundary/matching conditions are required in order to close the system of equations arising from the BEM sub-domain approach. The interface boundary conditions (IBCs) for a potential problem establish the continuity of the potential at every node at the interface, in this case node I, as shown in (10)

$$
u_{1}\left(r_{I}\right)=u_{2}\left(r_{I}\right)
$$

and the conservation of flux, as shown in (11)

$$
\Phi_{12}\left(u_{1}, q_{12}\right)=-\Phi_{21}\left(u_{2}, q_{21}\right)
$$

where the expression for the flux $\Phi$ in (11) depends on the physical problem under consideration. The situation with node $\mathbf{J}$ is more complicated, as this node is shared between four sub-domains. For homogeneous domains and when continuous boundary elements are used the IBCs yield to an over-determined system, which can be avoided by using discontinuous elements on the expense of increased number of degrees of freedom. 


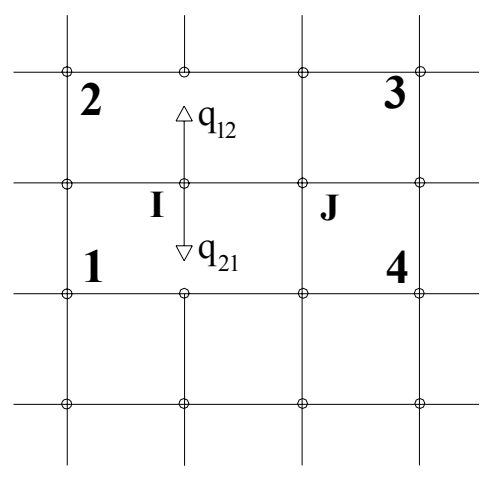

Figure 1: $\quad$ Example of sub-domains in a mesh.

\section{Overlapping BEM sub-domain technique}

The overlapping BEM sub-domain technique places the source point in the interior of the sub-domain. The main idea is to avoid placing the source on the boundary in order to avoid near-singular and/or singular integrals. In Figure 2 one can see two sub-domains, the first one being K-O-P-L with a source point in $\mathrm{M}$ and the second one being I-M-N-J with a source point in L, where the overlapping part of the two sub-domains is marked between points $\mathrm{M}$ and $\mathrm{L}$.

The proposed formulation solves in each interior node three integral equations in order to obtain the potential $u$, and the partial derivatives $\partial u / \partial x_{\mathrm{j}}$. The equations for derivatives $\partial u / \partial x_{\mathrm{j}}$ are obtained by differentiating equation (5) in respect to $x_{\mathrm{j}}$, where $x_{\mathrm{j}}$ are components of $r$.

The expression for partial derivatives is shown in (12), where the integrals are evaluated over the boundary $\Gamma_{\mathrm{i}}$ of the $i$-th sub-domain:

$$
\begin{aligned}
& \frac{\partial u(r)}{\partial x_{j}}=-\int_{\Gamma_{i}} \frac{\partial q^{*}(r, \xi)}{\partial x_{j}} u(\xi) d \Gamma_{\xi}+\int_{\Gamma_{i}} \frac{\partial u^{*}(r, \xi)}{\partial x_{j}} q(\xi) d \Gamma_{\xi}+ \\
& \sum_{k=1}^{J_{b n}+J_{i n}}\left\{\alpha_{k}\left(\frac{\partial \hat{u}\left(r, \eta_{k}\right)}{\partial x_{j}}+\int_{\Gamma_{i}} \frac{\partial q^{*}(r, \xi)}{\partial x_{j}} \hat{u}\left(\xi, \eta_{k}\right) d \Gamma_{\xi}-\int_{\Gamma_{i}} \frac{\partial u^{*}(r, \xi)}{\partial x_{j}} \hat{q}\left(\xi, \eta_{k}\right) d \Gamma_{\xi}\right)\right\}
\end{aligned}
$$

The discretized form of equation (12) for the unknown $\partial u / \partial x_{\mathrm{j}}$ at node $i$ is given as:

$$
\frac{\partial u_{i}}{\partial x_{j}}=\frac{\partial u_{i}}{\partial x_{j}}=-\sum_{k=1}^{J_{b n}} H_{i k, j} u_{k}+\sum_{k=1}^{J_{b n}} G_{i k, j} q_{k}+\sum_{k=1}^{J_{b n}+J_{i n}}\left\{\alpha_{k}\left(\sum_{m=1}^{J_{b n}} H_{i m, j} \hat{u}_{m k}-\sum_{m=1}^{J_{b n}} G_{i m, j} \hat{q}_{m k}+c_{i} \hat{u}_{i k}\right)\right\}
$$

where $H_{i k, j}$ and $G_{i k, j}$ are the influence coefficients obtained from the integrations of $\left(\partial q^{*} / \partial x_{j}\right)$ and $\left(\partial u^{*} / \partial x_{j}\right)$, respectively, at each boundary element. The normal derivative $q$ in the equation (13) can be written as: 


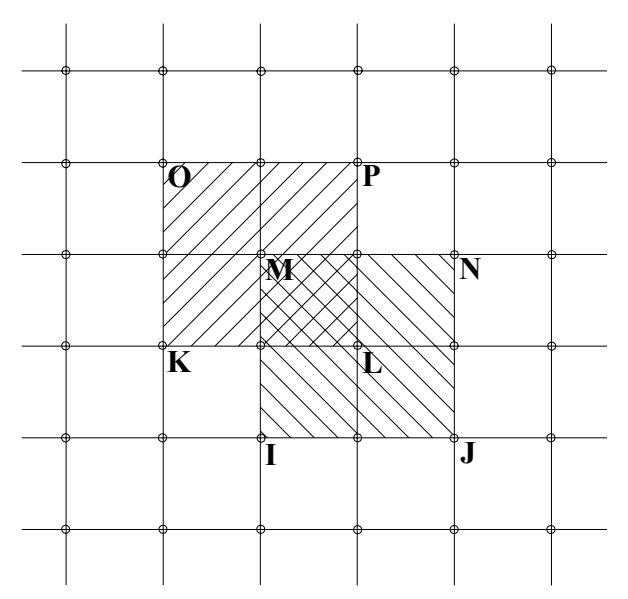

Figure 2: $\quad$ Part of the mesh used in the overlapping BEM sub-domain technique.

$$
q=\frac{\partial u}{\partial n}=\nabla u \cdot \vec{n}=\sum_{k} \frac{\partial u}{\partial x_{k}} n_{k}
$$

where $n_{k}$ are components of the unit normal vector. According to the above (5) can be written as:

$$
u_{i}+\sum_{j=1}^{J_{b n}} H_{i j} u_{j}-\sum_{m} \sum_{j=1}^{J_{b n}} G_{i j m} \frac{\partial u_{j}}{\partial x_{m}}=\sum_{k=1}^{J_{b n}+J_{i n}}\left\{\alpha_{k}\left(\sum_{j=1}^{J_{b n}} H_{i j} \hat{u}_{j k}-\sum_{j=1}^{J_{b n}} G_{i j} \hat{q}_{j k}+c_{i} \hat{u}_{i k}\right)\right\}
$$

where $G_{i j m}=G_{i j} n_{m}$. Substituting $q$ from (14) into (13) yields:

$$
\frac{\partial u_{i}}{\partial x_{j}}=-\sum_{m=1}^{J_{b n}} H_{i m, j} u_{m}+\sum_{p} \sum_{m=1}^{J_{b n}} G_{i m p, j} \frac{\partial u_{m}}{\partial x_{p}}+\sum_{k=1}^{J_{b n}+J_{i n}}\left\{\alpha_{k}\left(\sum_{m=1}^{J_{b n}} H_{i m, j} \hat{u}_{m k}-\sum_{m=1}^{J_{b n}} G_{i m, j} \hat{q}_{m k}+c_{i} \hat{u}_{i k}\right)\right\}
$$

where $G_{i m p, j}=G_{i m, j} n_{p}$.

\section{Solution procedures}

There are two solution procedures applied depending on whether the considered node is on the boundary or in the interior of the domain. 


\subsection{Interior nodes}

For interior nodes equations (15) and (16) are applied at each node. Since the potential and the partial derivatives are found from these two equations the system is closed and can be solved.

\subsection{Nodes on the boundary}

For nodes on the boundary the solution procedure applied by Natalini and Popov [2] has been applied.

When the source is in the interior and the integration is done over part of the boundary then instead of fluxes/normal derivatives, partial derivatives would be required in equations (15) and (16). They are obtained, as explained in [2], from the normal derivatives.

\section{Numerical examples}

The example used for verification of the approach is a 1-D Convection-Diffusion (C-D) problem with the following governing equation [3, 4]:

$$
D \frac{d^{2} u}{d x^{2}}-\frac{d\left(V_{x} u\right)}{d x}=0
$$

A rectangular domain with length $L$ and width $W$ is considered. In order to produce equivalent 1-D results in a 2-D domain the following BCs were applied:

$$
u(0, y)=U_{0} ; u(L, y)=U_{1} ;\left.\quad \frac{\partial u}{\partial y}\right|_{y=W / 2}=\left.\frac{\partial u}{\partial y}\right|_{y=-W / 2}=0
$$

The velocity field is defined as:

$$
V_{x}=\ln \frac{U_{1}}{U_{0}}+k\left(x-\frac{1}{2}\right) ; \quad V_{y}=0 .
$$

The analytical solution of the above problem for $D=1 \mathrm{~m}^{2} \mathrm{~s}^{-1}$ is given by:

$$
u(x)=U_{0} \exp \left\{\frac{k}{2} x^{2}+\left(\ln \frac{U_{1}}{U_{0}}-\frac{k}{2}\right) x\right\}
$$

The numerical results are obtained using the augmented thin plate spline (ATPS) [5] and $f=1+R[1]$ as DRM approximation functions. The results of the present approach are compared to the analytical results and the numerical ones obtained by the DRM-MD by Popov and Power [4].

In this example, three different meshes are generated in order to test the accuracy and the convergence of the method. The first mesh is generated with 95 nodes and 119 sub-domains. The second mesh is generated with 185 nodes and 229 sub-domains and is shown in Figure 3. The third mesh has 365 nodes and 449 sub-domains as shown in Figure 4. Two interior and two sub-domains on the boundary are marked with different colors. For each mesh two values of the parameter $k(k=10$ and $k=40)$ and two approximation functions, the ATPS and $f=1+R$ are considered. The maximum velocity, equivalent to the Peclet number since $\mathrm{D}=\mathrm{L}=1$, reach values 8.4 for $k=10$ and 23.4 for $k=40$. 


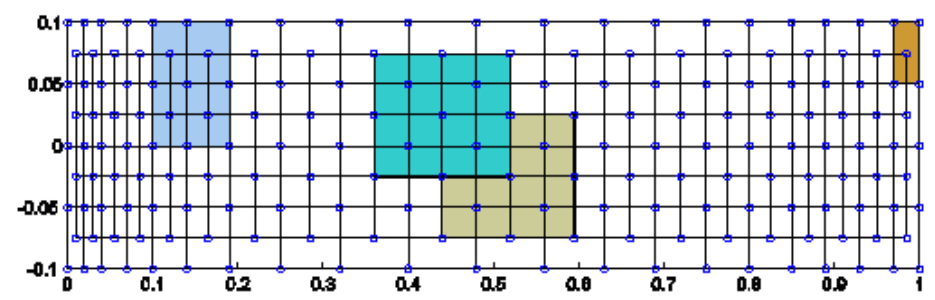

Figure 3: $\quad$ Mesh with 185 nodes used for the C-D problem.

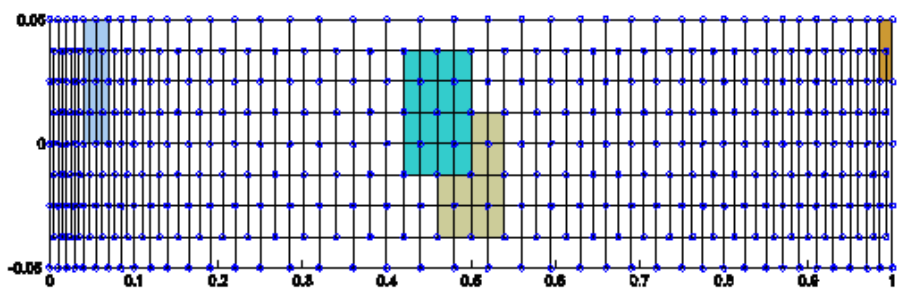

Figure 4: $\quad$ Mesh with 365 nodes used for the C-D problem.

The relative errors for the potential for the case $k=40$ are plotted in Figure 5 for the ATPS and in Figure 6 for $f=1+R$. As can be seen in the figures the relative error decreases with the increase of number of nodes in the mesh, which confirms the convergence of the method. The coarse mesh with 95 nodes with ATPS had a maximum error of approximately $200 \%$ which is not shown in Figure 5.

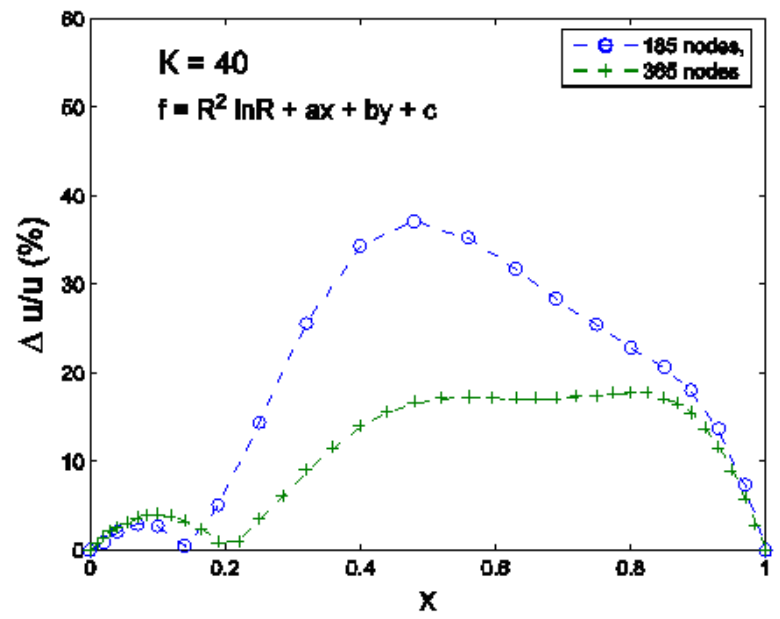

Figure 5: Relative error for the potential for the C-D problem (case $K=40$, approximation function ATPS). 
186 Boundary Elements and Other Mesh Reduction Methods XXX

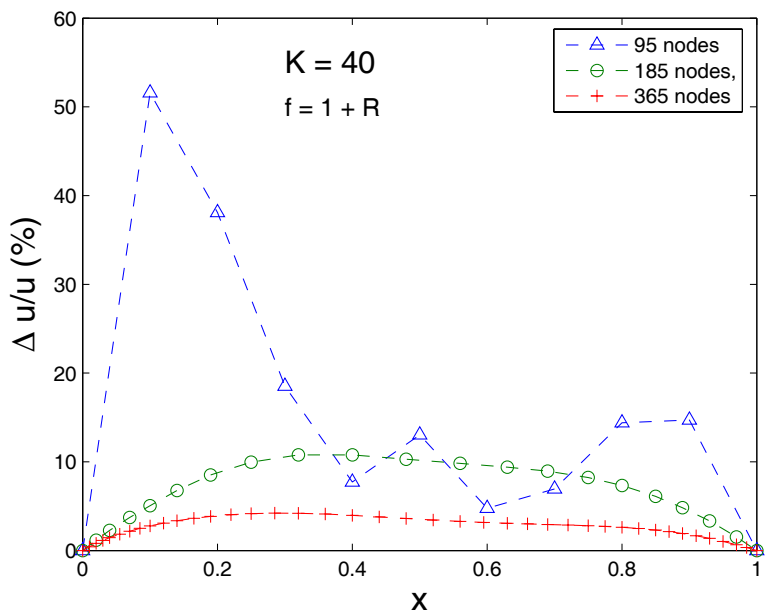

Figure 6: Relative error for the potential for the C-D problem (case $K=40$, approximation function $f=1+R$ ).

Table 1: $\quad$ Relative error for the potential values at internal points obtained using the present method and the DRM-MD approach [4] for $k=40$.

\begin{tabular}{|r|r|r|r|}
\hline \multicolumn{1}{|c|}{$x$} & \multicolumn{1}{|c|}{ DRM-MD } & $f=1+r$ & \multicolumn{1}{l|}{ ATPS } \\
\hline 0.00 & 0.00000 & 0.00000 & 0.00000 \\
\hline 0.02 & 1.60078 & 1.16151 & 0.93093 \\
\hline 0.04 & 2.88296 & 2.26563 & 2.04848 \\
\hline 0.07 & 5.16544 & 3.73569 & 2.93691 \\
\hline 0.10 & 6.83983 & 5.05555 & 2.72869 \\
\hline 0.14 & 9.38016 & 6.76309 & 0.48519 \\
\hline 0.19 & 11.82482 & 8.51887 & 5.10446 \\
\hline 0.25 & 13.46586 & 9.95951 & 14.41637 \\
\hline 0.32 & 13.72012 & 10.76810 & 25.54530 \\
\hline 0.40 & 12.41174 & 10.77337 & 34.33515 \\
\hline 0.56 & 8.52606 & 10.46788 & 35.25292 \\
\hline 0.75 & 3.98747 & 8.23023 & 25.47853 \\
\hline 0.85 & 0.69131 & 6.09553 & 20.63368 \\
\hline 0.93 & 0.52574 & 3.33171 & 13.70113 \\
\hline 1.00 & 0.00000 & 0.00000 & 0.00000 \\
\hline & & & \\
\hline
\end{tabular}


In Table 1 the errors for the potential obtained by applying the DRM-MD and the proposed approach with the mesh with 185 and the two approximation functions are shown. It appears that the proposed approach with the approximation function $f=1+R$ produces better results than the other two formulations.

\section{Conclusions}

An overlapping BEM sub-domain technique has been developed with two different DRM approximation functions, ATPS and $f=1+R$. The proposed approach reduces some of the problems introduced by sub-domain techniques in the BEM, e.g., IBCs, which can introduce significant difficulties when implementing the numerical scheme, as well as singularities in the integrals arising from the formulation itself or distorted sub-domains due to complex geometries.

The formulation has been tested on a convection-diffusion problem with variable velocity for three different meshes with 95 nodes, 185 and 365 showing good accuracy and convergence with increasing refinement of the mesh. The approximation function $f=1+R$ has shown better accuracy than the ATPS.

The results obtained with the proposed approach have been compared to the DRM-D results obtained previously by Popov and Power [4], and showed higher accuracy than the DRM-MD when $f=1+R$ is used.

The proposed approach has been developed as an intermediate step towards a meshless integral equation approach which will be reported in the near future.

\section{References}

[1] Partridge, P.W., Brebbia C.A., Wrobel L.C. The dual reciprocity boundary element method, Southampton: Elsevier Applied Science; 1992.

[2] Natalini, B., Popov, V. (2004), An alternative approach for calculation of the first and higher order derivatives in the DRM-MD, Engineering Analysis with Boundary Elements, 28/1, 61-78.

[3] DeFigueiredo, D. B. Boundary Element analysis of conection-diffusion problems, Ph.D. Thesis, Wessex Institute of Technology, Southampton, U.K. 1990.

[4] Popov, V., and Power, H. The DRM-MD integral equation method: an efficient approach for the numerical solution of domain dominant problems, Int. J. Numer. Meth. Eng., 44 (1999) 327-353.

[5] Golberg MA, Chen CS, The theory of radial basis functions applied to the BEM for inhomogeneous partial differential equations, Bound Elem Commun 1994; 5:57-61. 